\title{
Anti-Mullerian Hormone Changes Following Laparoscopic Ovarian Cystectomy: A Prospective Comparative Study
}

\author{
Hye-yon Cho \\ Sung-taek Park \\ Sung-ho Park \\ Min Sun Kyung (D) \\ 'Department of Obstetrics \& \\ Gynecology, Hallym University Dongtan \\ Sacred Heart Hospital, Hwaseong, Korea; \\ ${ }^{2}$ Department of Obstetrics \& \\ Gynecology, Hallym University Gangnam \\ Sacred Heart Hospital, Seoul, Korea
}

Correspondence: Min Sun Kyung

Department of Obstetrics and

Gynecology, Hallym University Medical

Center, 40 Seoku-dong, Hwaseong,

I8450, Korea

Tel +82-3I-8086-2898

Fax +82-3I-8086-2650

Email msfeel@hallym.or.kr
Purpose: To investigate whether laparoscopic ovarian cystectomy (LOC) affects ovarian reserve.

Patients and Methods: In 46 premenopausal women, who underwent either LOC (study group, $\mathrm{n}=26$ ) or laparoscopic myomectomy (LM) (control group, $\mathrm{n}=20$ ), serum antiMullerian hormone (AMH) levels were measured pre-operatively (AMH0), and postoperatively at 7 days (AMH1), 2 months (AMH2), and 6 months (AMH3). Changes in AMH from baseline level (AMH0) in each group were compared.

Results: AMH0 did not differ between the two groups $(3.5 \pm 3.33$ in LOC vs $2.4 \pm 2.72$ in LM, $P=0.250$ ). AMH1, $\mathrm{AMH} 2$, and $\mathrm{AMH} 3$ in each group were also similar. However, a significant decline of $\mathrm{AMH}$ (ie more than $50 \%$ decrease compared to AMH0) at postoperative 6 months occurred more frequently in the LOC group than in the LM group. In the sub-analysis of the LOC group, a significant decline of AMH at postoperative 2 months and 6 months was highly correlated with bilateral ovarian tumors $(P=0.001)$.

Conclusion: Compared to LM, serum AMH level showed a minimal decrease after 1 week following LOC, which did not revert to normal over 6 months of follow-up. In addition, a significant decline of ovarian reserve at postoperative 6 months was significantly more frequent in the LOC group, suggesting that LOC may have more adverse effects on ovarian reserve compared to the LM (control) group. Thus, care is required during the LOC procedure, specifically in women with bilateral tumors.

Keywords: AMH, laparoscopy, ovarian cystectomy, ovarian reserve, electrocautery

\section{Introduction}

Laparoscopic ovarian cystectomy (LOC) is one of the most widely used fertilitypreserving procedures used in gynecologic surgery. Ovarian tumors are common in women of all ages, and up to $10 \%$ of those women undergo surgical removal because of the risk of ovarian malignancy or of complications, such as ovarian cyst hemorrhage, rupture, and torsion. ${ }^{1,2}$ Surgical removal can be achieved by various procedures, such as aspiration, cystectomy, and partial or complete oophorectomy. Usually, LOC is preferred for women who desire to preserve fertility. ${ }^{2}$ LOC allows complete excision of the cyst wall (by the so-called stripping method), and preservation of healthy ovarian tissue. However, the safety to the patient of this procedure remains contentious because of its potential risk of causing decreased ovarian reserve and premature ovarian failure. Indeed, several reports have suggested that ovarian reserve, as measured by serum AMH levels, decreased following LOC in women with endometrioma. ${ }^{3-6}$ 
AMH is expressed by the granulosa cells of active growing ovarian follicles from their initial recruitment to cyclic selection and it negatively regulates primordial follicle recruitment. ${ }^{7,8} \mathrm{AMH}$ is considered to be a reliable marker for ovarian reserve. ${ }^{9-11}$ AMH gradually declines with increasing age and is independent of the ovarian cycle. ${ }^{12,13}$ Moreover, AMH level is correlated with other markers of ovarian reserve, such as an antral follicle count of cycle day 3 serum FSH concentration, and can predict ovarian responsiveness to hyperstimulation, or impending menopausal transition. ${ }^{8,14,15}$ In clinical practice, the serum AMH is widely used to estimate ovarian reserve in women with suspicious menopausal symptoms or in infertile women who wish to undergo assisted reproductive technology (ART). ${ }^{15}$ Several studies have reported on the use of serum AMH to evaluate ovarian reserves following procedures such as the excision of ovarian endometrioma, and hysterectomy. ${ }^{12,15,16}$

Nonetheless, only a few reports have described the long-term effects of LOC on residual ovarian function and the results have been equivocal. ${ }^{17-19}$ Furthermore, most prior studies have been of infertile women with ovarian endometrioma, with a control group that was not homogeneous. ${ }^{17-19}$ There might be some differences between women with endometriomas and other benign ovarian cysts in relation to the decrease of ovarian reserve following LOC.

Therefore, we aimed to evaluate the ovarian reserves of women who underwent LOC compared to those women who underwent non-ovarian surgery, by taking serial measurements of the serum AMH level over 6 months of follow-up.

\section{Patients and Methods}

For this prospective study, we recruited patients who underwent laparoscopic cystectomy for benign ovarian disease and laparoscopic myomectomy, at Hallym University Medical Center, Korea, between January 2016 and December 2018. The inclusion criteria were 1) women who were diagnosed with benign ovarian tumor or uterine myoma by ultrasound, 2) who underwent laparoscopic ovarian cystectomy (the study group) or laparoscopic myomectomy (the control group), and 3) who checked serum AMH at preoperatively, and postoperative 7 days, 2 months, and 6 months. The exclusion criteria were 1) postmenopausal women, 2) a prior history of adnexal surgery, 3) hormonal treatment within 3 months of surgery, 4) women undergoing both LOC and LM, and 5) co-existence of endocrine disease.

During the follow-up period (postoperative 6 months), no women were treated with hormonal therapy.

This study was approved by the local institutional review board and all recruited patients provided informed written consent. This study was conducted in accordance with the Declaration of Helsinki.

\section{Sample Size Calculation}

The study aimed to show 1.0 difference in mean AMH between the two groups. Standard deviation was assumed to be similar in both groups. Based on two-tailed power calculation with $80 \%$ power, 0.05 alpha, $1: 1$ allocation, 26 patients needed to be included in the trial to show this difference.

\section{Surgical Technique}

Each laparoscopic procedure was performed by a senior surgeon (there were four in all), each of whom had performed over 200 LOC and LM procedures.

The LOC technique was as follows. After a careful incision of the ovarian cyst surface using monopolar scissors (Karl Storz Monopolar Forceps ${ }^{\mathrm{TM}}$, Karl Storz, Tuttlingen, Germany), the ovarian cyst wall was dissected and completely removed from the ovarian cortex by the traction/counter traction method with non-traumatic grasping forceps. Bleeding control, using laparoscopic bipolar forceps (Karl Storz Bipolar Forceps ${ }^{\mathrm{TM}}$, Karl Storz, Tuttlingen, Germany) and the Force FX ${ }^{\mathrm{TM}}$ Electrosurgical Generator (Valleylab) with 35 watt setting was careful and highly selective. Suturing of the residual ovarian cortex was not routinely performed. The enucleated ovarian cyst was placed in a retrieval bag (Lap bag ${ }^{\mathrm{TM}}$, Sejong Medical, Seoul, Korea) and extracted through the $12 \mathrm{~mm}$ trocar site.

The LM technique was as follows. After an injection of a dilute solution of vasopressin (10 IU/100mL normal saline) into the tissue adjacent to the base and capsule of the uterine myoma, an incision was made into the myometrium, using monopolar scissors. With careful dissection and coagulation, the uterine myoma was detached. Then, the uterine muscle layer was repaired with either 1 or 2 layers of continuous suturing (depending on the depth of the wound) using unidirectional barbed suture material (1-0 V-Loc $180^{\mathrm{TM}}$, Covidien, Mansfield, MA, USA). Detached myomas were placed in a retrieval bag and extracted through the 11-mm trocar site with a $12 \mathrm{~mm}$ electronic morcellator (Rotocut $^{\mathrm{TM}}$ G1 Morcellator, Karl Storz, Tuttlingen, Germany). 


\section{Hormonal Assay}

Serum AMH levels were measured in each group as follows: AMH0 (preoperatively, within 2 weeks prior to surgery); AMH1 (postoperative day 7); AMH2 (postoperative 2 months); and AMH3 (postoperative 6 months). Although AMH levels are well known to not be affected by hormonal periods, blood sampling was done at 7 th to 10th day of menstrual period. Therefore, AMH sampling was done. We used commercially available enzyme-linked immunosorbent assay kits (Diagnostic System Laboratories Inc., Webster, TX) to measure serum AMH levels. Serum AMH levels were reported as nanograms per milliliter with a detection limit of $0.006 \mathrm{ng} / \mathrm{mL}$.

\section{Statistical Analysis}

For all statistical analysis, SPSS for Windows (version 22.0, SPSS Inc., Chicago, Illinois) was used. Continuous variables were compared using the $t$-test. Dichotomous variables were compared using Fisher's exact test or the chi-square test. A $P$ value less than 0.05 was considered to be significant for all statistical tests.

\section{Results}

A total of 46 patients $(\mathrm{LOC}=26, \mathrm{LM}=20)$ who agreed to participate were included in the study. Of those, 30 patients continued in the study until the last follow-up visit at 6 months. Their mean age was 36.8 years.

The baseline data of all patients is presented in Table 1. Patients in the LOC group were significantly younger than those in the LM group, and the mean BMI was significantly lower in the LM group than in the LOC group. Parity was not different between the two groups. Among the 26 women in LOC group, 5 were confirmed (by pathology) with endometriomas, and the other 21 as nonendometriomas. The details of the pathology of the LOC group are described in Table 2.

Changes of serum AMH after surgery are shown in Table 3. AMH0 was similar in the two groups $(3.5 \pm 3.33$ in $\mathrm{LOC}$ vs $2.4 \pm 2.72$ in $\mathrm{LM}, P=0.250$ ). AMH1, $\mathrm{AMH} 2$,

Table I Baseline Data

\begin{tabular}{|l|c|c|c|}
\hline & LOC $\mathbf{( N = 2 6 )}$ & LM $(\mathbf{N}=20)$ & $P$ value \\
\hline Age (years) & $33.5 \pm 8.73$ & $41.3 \pm 5.77$ & $0.00 I^{*}$ \\
Parity & $\mathrm{I} \pm 0.99$ & $\mathrm{I} \pm 1.03$ & 0.174 \\
BMI & $21.5 \pm 3.29$ & $24.9 \pm 4.4 \mathrm{I}$ & $0.004^{*}$ \\
\hline
\end{tabular}

Notes: Baseline data are presented. $* P$ value $<0.05$. Data are presented as mean \pm SD or median \pm SEM.
Table 2 Pathology of Women with Ovarian Cystectomy

\begin{tabular}{|l|l|}
\hline Pathology & $\mathbf{N}=\mathbf{2 6}$ \\
\hline Endometriosis & $4(I 5.4)$ \\
Mature cystic teratoma & $8(30.8)$ \\
Mucinous cystadenoma & $4(15.4)$ \\
Abscess & $\mathrm{I}(3.8)$ \\
Follicular cyst & $3(11.5)$ \\
Adenofibroma & $5(19.2)$ \\
Endometriosis \& adenofibroma & $\mathrm{I}(3.8)$ \\
\hline
\end{tabular}

Notes: Pathology of women with ovarian cystectomy are presented. Data was presented as number (\%).

and AMH3 were also similar in both groups. However, a significant decline of AMH (ie more than $50 \%$ decrease compared to AMH0) was more frequently observed after 6 months of surgery in the LOC group than in the LM group. The serial changes of serum AMH levels after LOC and LM are depicted in Figure 1. The serum AMH level, which was barely changed in LM group, abruptly decreased after 1 week of surgery and did not recover to pre-operative levels even in the 6 months after surgery in the LOC group; however, there were no statistically significant differences between AMH0 and AMH3 levels.

An older age was a clinical characteristic associated with a significant decline of AMH after postoperative 6 months ( $\geq 39$ yrs.; $P=0.035$ ) (Table 4 ).

In the sub-analysis of the LOC group, a significant decline of AMH was highly correlated with bilateral ovarian tumor $(P=0.001)$. Age, BMI, and tumor size were not correlated with a significant change in AMH (Table 5).

\section{Discussion}

Currently, laparoscopic ovarian cystectomy is considered to be the primary treatment of choice for benign ovarian tumors. ${ }^{20}$ The main benefit is thought to be a more complete excision of the tumor during the procedure. ${ }^{21}$ Nonetheless, reduction of residual ovarian function and increased risk of premature ovarian failure induced by an excessive removal of healthy ovarian tissue remains an issue. $^{3,4,21}$ Indeed, there is an accumulation of evidence indicating that laparoscopic ovarian cystectomy for the treatment of benign tumor in premenopausal women has a negative impact on ovarian reserve. ${ }^{15,18,21,22}$

Most researchers agree that ovarian reserve, as represented by the patient's serum AMH level, is slightly or significantly decreased 1 to 3 months after surgery. ${ }^{18,23,24}$ A prospective study evaluating ovarian reserve after cystectomy for ovarian endometriosis revealed 
Table 3 Changes in Serum AMH Levels Following LOC and LM

\begin{tabular}{|l|c|c|c|}
\hline & LOC (N=26) & LM (N=20) & P value \\
\hline AMH levels & & & 0.250 \\
Preoperative (AMH0) & $3.5 \pm 3.33$ & $2.4 \pm 2.72$ & 0.788 \\
Postoperative 7 days (AMHI) & $2.4 \pm 2.46$ & $2.6 \pm 3.28$ & 0.957 \\
Postoperative 2 months (AMH2) & $2.6 \pm 2.83$ & $2.6 \pm 3.11$ & 0.414 \\
Postoperative 6 months (AMH3) & $1.6 \pm 2.23$ & $2.6 \pm 3.60$ & 0.079 \\
\hline AMH changes comparing to preoperative level & & & $0.19 \pm 1.44$ \\
Postoperative 7 days (AMHI) & $-1.1 \pm 2.98$ & $0.1 \pm 1.32$ & 0.136 \\
Postoperative 2 months (AMH2) & $-0.9 \pm 3.20$ & $0.0 \pm 1.81$ & 0.332 \\
Postoperative 6 months (AMH3) & $-1.7 \pm 4.34$ & $2(10.0)$ & 0.547 \\
AMHI decrease $\geq 50 \%$ (N=7/46) & $5(19.2)$ & $4(20.0)$ & $0.00 I^{*}$ \\
AMH2 decrease $\geq 50 \%$ (N=10/46) & $6(23.1)$ & $4(21.0)$ & \\
AMH3 decrease $\geq 50 \%(\mathrm{~N}=8 / 30)$ & $4(36.4)$ & & \\
\hline
\end{tabular}

Notes: Changes in serum AMH levels following LOC and LM were presented. *P value $<0.05$.

a temporary decreased serum AMH level, which reverted to normal within 12 months of follow-up. ${ }^{19}$ However, there are very few reports of long-term follow-up and it remains controversial as to whether the ovarian reserve is restored within 6 months to 1 year following surgery. 5,17,18,23,25-27
According to one prospective follow-up study of 71 women who underwent ovarian cystectomy (21 bilateral ovarian endometriomas, 29 unilateral ovarian endometriomas, and 21 unilateral other benign ovarian cysts), serum AMH level was significantly decreased at postoperative 1 month, but restored within 1 year. ${ }^{18}$ Another

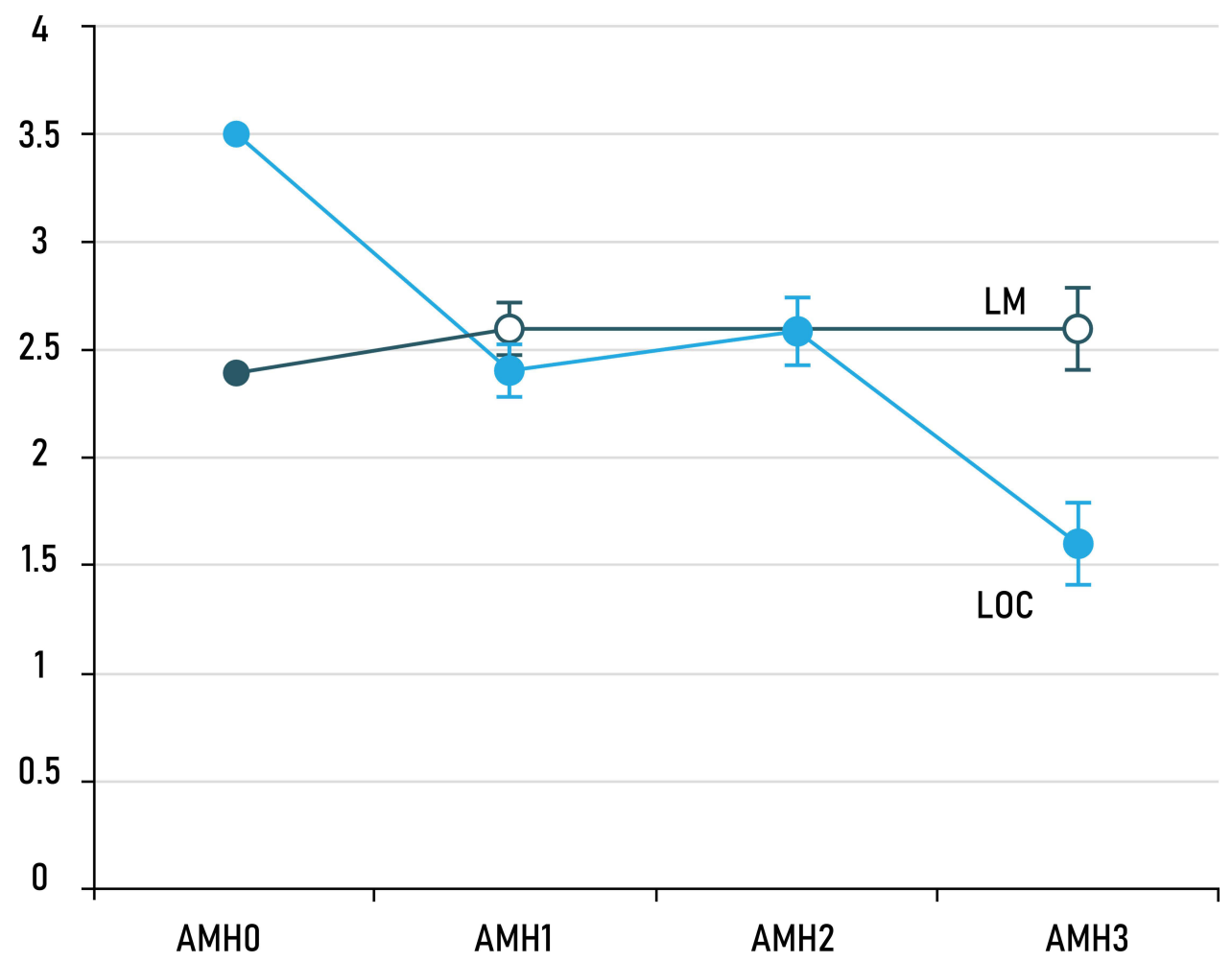

Figure I Serial changes of serum AMH levels following LOC and LM. The serum AMH level, which barely changed in the LM group, abruptly decreased in the LOC group I week after surgery and had not recovered to pre-operative levels 6 months after surgery. However, in this group there were no statistically significant differences between $\mathrm{AMHO}$ and $\mathrm{AMH} 3$. 
Table 4 Correlation Between Changes in AMH and Clinical Characteristics

\begin{tabular}{|c|c|c|c|c|c|c|}
\hline & $\begin{array}{c}\text { AMHI Decrease } \geq 50 \% \\
(N=7 / 46)\end{array}$ & $P$-value & $\begin{array}{c}\text { AMH2 Decrease } \geq 50 \% \\
(N=10 / 46)\end{array}$ & $P$-value & $\begin{array}{c}\text { AMH3 Decrease } \geq \mathbf{5 0 \%} \\
(\mathrm{N}=\mathbf{8} / \mathbf{3 0})\end{array}$ & $P$-value \\
\hline $\begin{array}{l}\text { Age } \\
\qquad \begin{array}{l}<39 \mathrm{yrs} \\
\geq 39 \mathrm{yrs}\end{array}\end{array}$ & $\begin{array}{l}3(13.0) \\
4(17.4)\end{array}$ & 0.500 & $\begin{array}{l}3(13.0) \\
7(30.4)\end{array}$ & 0.142 & $\begin{array}{l}2(8.7) \\
6(26.1)\end{array}$ & $0.035 *$ \\
\hline $\begin{array}{l}\text { BMI } \\
\quad<20 \\
\geq 20\end{array}$ & $\begin{array}{l}3(27.3) \\
4(11.4)\end{array}$ & 0.207 & $\begin{array}{l}5(14.3) \\
5(21.7)\end{array}$ & $0.043^{*}$ & $\begin{array}{l}3(27.3) \\
5(14.3)\end{array}$ & 0.544 \\
\hline
\end{tabular}

Notes: Correlation between changes in $\mathrm{AMH}$ and clinical characteristics was presented. ${ }^{*} P$ value $<0.05$.

Table 5 Correlation Between Changes in AMH and Clinical Characteristics Following LOC

\begin{tabular}{|c|c|c|c|c|c|c|}
\hline & $\begin{array}{l}\text { AMHI Decrease } \geq 50 \% \\
(N=5 / 26)\end{array}$ & $P$-value & $\begin{array}{c}\text { AMH2 Decrease } \geq 50 \% \\
(N=6 / 26)\end{array}$ & $P$-value & $\begin{array}{c}\text { AMH3 Decrease } \geq 50 \% \\
(N=4 / I I)\end{array}$ & $P$-value \\
\hline $\begin{array}{l}\text { Age } \\
\qquad \begin{array}{l}<39 \mathrm{yrs} \\
\geq 39 \mathrm{yrs}\end{array}\end{array}$ & $\begin{array}{l}3(17.6) \\
2(22.2)\end{array}$ & 0.580 & $\begin{array}{l}3(17.6) \\
3(33.3)\end{array}$ & 0.332 & $\begin{array}{l}2 \text { (I I.8) } \\
2(22.2)\end{array}$ & 0.182 \\
\hline $\begin{array}{l}\text { BMI } \\
\qquad 20 \\
\geq 20\end{array}$ & $\begin{array}{l}2(22.2) \\
3(17.6)\end{array}$ & 0.580 & $\begin{array}{l}4(44.4) \\
2(11.8)\end{array}$ & 0.084 & $\begin{array}{c}3(33.3) \\
1(5.9)\end{array}$ & 0.105 \\
\hline $\begin{array}{l}\text { Bilateral tumor } \\
\text { No } \\
\text { Yes }\end{array}$ & $\begin{array}{l}3(15.8) \\
2(28.6)\end{array}$ & 0.411 & $\begin{array}{l}2(10.5) \\
4(57.1)\end{array}$ & $0.028 *$ & $\begin{array}{c}0(0.0) \\
4(57.1)\end{array}$ & $0.00 I^{*}$ \\
\hline $\begin{array}{l}\text { Tumor diameter } \\
\quad<7 \mathrm{~cm} \\
\geq 7 \mathrm{~cm}\end{array}$ & $\begin{array}{l}2(11.1) \\
3(37.5)\end{array}$ & 0.150 & $\begin{array}{l}4(22.2) \\
2(25.0)\end{array}$ & 0.622 & $\begin{array}{l}4(22.2) \\
0(0.0)\end{array}$ & 0.302 \\
\hline
\end{tabular}

Notes: Correlation between changes in AMH and clinical characteristics following LOC was presented. $* P$ value $<0.05$.

prospective study of 22 women with endometrioma (10 with unilateral tumors and 12 with bilateral tumors) reported that serum AMH levels were significantly decreased at postoperative 1,3 , and 6 months but were completely restored within 1 year of follow-up. ${ }^{17}$ Moreover, women with bilateral endometriomas had a significantly higher rate of decline in AMH levels at postoperative 1 year than those with unilateral endometriomas. ${ }^{17}$ Furthermore, several studies have reported that ovarian reserve is not restored after 6 months to 1 year. ${ }^{23,28-30}$ According to an observational study of 25 women with unilateral endometrioma, serum AMH level was decreased by $24 \%$ of the baseline level at postoperative 1 month and was not restored until postoperative 6 months. ${ }^{29}$ Another prospective study of 30 women with endometrioma reported a minimal decline in AMH levels at postoperative 1 month, which then further significantly declined until postoperative 6 months. ${ }^{26}$ Our results are in accordance with these reports. The AMH level minimally decreased after 7 days, 1 month, and 2 months of surgery. Although the serum AMH levels at postoperative 6 months were not significantly different from those of the baseline, $36.4 \%$ of women who underwent LOC showed a significant decline of ovarian reserve (ie more than $50 \%$ decrease from baseline) at postoperative 6 months. Interestingly, this permanent damage to the ovary tissue can affect future pregnancies in infertile women. According to a prospective study of 54 infertile women who underwent ovarian cystectomy for the treatment of endometrioma, the postoperative 1-year serum AMH level may be a possible predictor for the likelihood of pregnancy. ${ }^{28}$ In that study, 17 women became pregnant, and the serum AMH levels 1-year postoperatively were significantly higher in the pregnant group compared to the non-pregnant group $(3.44 \pm 1.78$ versus $2.17 \pm 2.24$ $\mathrm{ng} / \mathrm{mL}, P=0.049){ }^{28}$ 
However, most past studies have been of infertile women with endometrioma. It remains unclear, due to lack of data, as to whether the impact of LOC on ovarian reserve is similar between women with or without endometrioma, and between infertile women and healthy women. A recent study comparing women with endometrioma and healthy controls showed that women with endometrioma had lower serum AMH levels compared to the healthy controls. ${ }^{23}$ After surgical excision of endometrioma, their serum AMH levels further declined, which appeared permanent. ${ }^{23}$ Undertaking cystectomy in ovarian endometriomas which do not present with real cyst capsules presents a risk of possible inadvertent removal of healthy ovarian tissue. ${ }^{18}$ In addition, serum AMH levels in infertile women may differ from those in women with a normal menstruation cycle. In a prospective study of 27 women with polycystic ovarian syndrome (PCOS), which is a major cause of infertility, serum AMH levels were significantly higher in those women with PCOS compared to healthy controls. ${ }^{31}$ Also, the authors suggested that a higher serum AMH level, which represents the disordered folliculogenesis characteristic of PCOS, may be a useful predictive marker for persistent or recurrent disease. ${ }^{31}$

Our study investigated the impact on ovarian reserve of LOC undertaken for the treatment of benign ovarian tumors, by comparing serial changes of serum AMH levels with a homogeneous control group (LM) during 6 months of postoperative follow-up. Our data showed that ovarian reserve following LOC abruptly decreased at postoperative 7 days, although this was not significant, and this decreased serum AMH was sustained until postoperative 6 months, a finding similar to that of prior studies. ${ }^{18,26,30}$ By contrast, there were no abrupt changes of ovarian reserve following LM during the study period. Interestingly, a significant decline of ovarian reserve (ie more than $50 \%$ of decrease from baseline) at postoperative 6 months was more frequently observed in the LOC group compared to the LM group. The significant decline of ovarian reserve at postoperative 6 months was highly associated with an older age ( $\geq 39$ years). Considering that the mean age of the LM group was older than that of the LOC group, the decline of the AMH level after LOC seems clinically more significant. Therefore, care exercised in the operative procedure will be important for women of an older age.

In our sub-analysis of the LOC group, bilateral tumor was highly associated with a significant decline of ovarian reserve (more than $50 \%$ of decrease from baseline) at postoperative 2 months and 6 months, which is in accordance with findings of prior studies. ${ }^{25,32,33}$ However, age, BMI, and tumor size were not correlated with a significant decline of ovarian reserve, which is also similar to the findings of prior studies. ${ }^{17,30}$

Based on our results, we believe the possible mechanisms of decreased ovarian reserve after LOC are 1) the decline of ovarian volume during the stripping method; and 2) thermal damage to healthy ovarian tissue occurring during hemostasis with a bipolar device.

Many investigators have suggested that the inadvertent removal of adjacent healthy ovarian tissue during the stripping method is the possible main cause of decreased ovarian reserve after ovarian cystectomy. ${ }^{25,32,34,35}$ One retrospective study revealed that the stripping procedure employed in $90 \%$ of cases in both endometrioma and non-endometrioma groups removed normal ovarian tissue; and the tissue loss was greater when endometriotic cysts were involved. ${ }^{32,35}$ The correlation of hemostatic methods and ovarian reserve, however, is controversial. One prospective study of 80 women with unilateral or bilateral endometriomas reported that the serum AMH level decreased significantly from baseline to 6 months and 12 months after surgery, even with the ovarian suturing method. ${ }^{36}$ Other randomized controlled trials comparing differing techniques of laparoscopic ovarian cystectomy have revealed that a hemostatic suture group had no statistically significant change from baseline AMH levels $(P=0.165)$. By contrast, postoperative AMH levels in the bipolar electrocoagulation group were lower than baseline AMH levels $(P=0.028)$. Nonetheless, pregnancy rates were not significantly different $(P=0.546)$, and ovarian reserve did not decrease further during the follow-up period of up to 12 months following surgery. ${ }^{37}$ However, another prospective comparative study of 125 women with endometriomas undergoing laparoscopic-endoscopic single-site cystectomy reported that hemostasis using bipolar coagulation reduced ovarian reserve more than suturing. ${ }^{38}$ In addition, a meta-analysis comparing suture, hemostatic sealant, and use of a bipolar device revealed that suture and hemostatic sealant are significantly associated with the protection of residual ovarian function compared to bipolar coagulation. ${ }^{38}$ In our study, we used a bipolar device for hemostasis, which can be a major cause of reduced ovarian reserve even with a careful and selective coagulation.

There are some limitations to our study. First, our sample size was small and there was follow-up loss of some patients at postoperative 6 months. Second, ours was a prospective study rather than a randomized clinical trial. Nonetheless, our study has the advantages of having a homogeneous control group and a large portion of non-endometrioma women in the study group. Moreover, our findings are consistent with prior published results. ${ }^{18,26,30}$ 


\section{Conclusion}

Our results suggest that overall changes in ovarian reserve following LOC are minimal. However, if there are any, then the adverse post-LOC effect on ovarian reserve seems more significant than that following non-ovarian surgery. In addition, the compromised ovarian reserve is not restored until 6 months following LOC. Therefore, to avoid loss of ovarian reserve, more careful and highly selective hemostasis, more cautious preservation of ovarian volume, and less damage to the healthy ovarian tissue are prerequisites during the LOC procedure, particularly in the treatment of bilateral ovarian tumors. In addition, these negative impact on ovarian reserve should be informed to women who desire for future pregnancy or are concerned about early menopause.

Further large-scale prospective studies are required to examine the long-term outcomes of AMH level after surgery.

\section{Abbreviations}

AMH, anti-Mullerian hormone; ART, assisted reproductive technology; LM, laparoscopic myomectomy; LOC, laparoscopic ovarian cystectomy.

\section{Ethics Approval and Consent to Participate}

This study was approved by the local institutional review board of Hallym University Hospital (IRB No. 2016-0041). All patients were informed about the study verbally and in writing (with documentation), and participated in the study after providing written consent.

\section{Funding}

This research was supported by Hallym University Research Fund 2016(HURF-2016-23).

\section{Disclosure}

The authors report no conflicts of interest in this work.

\section{References}

1. Bottomley C, Bourne T. Diagnosis and management of ovarian cyst accidents. Best Pract Res Clin Obstet Gynaecol. 2009;23(5):711-724. doi:10.1016/j.bpobgyn.2009.02.001

2. Sisodia RM, Del Carmen MG, Boruta DM. Role of minimally invasive surgery in the management of adnexal masses. Clin Obstet Gynecol. 2015;58(1):66-75. doi:10.1097/GRF.0000000000000086

3. Ozgonen H, Erdemoglu E, Gunyeli I, Guney M, Mungan $T$. Comparison of the effects of laparoscopic bipolar electrocoagulation and intracorporeal suture application to ovarian reserve in benign ovarian cysts. Arch Gynecol Obstet. 2013;287(4):729-732. doi:10.1007/s00404-012-2638-2
4. Kwon SK, Kim SH, Yun SC, et al. Decline of serum antimullerian hormone levels after laparoscopic ovarian cystectomy in endometrioma and other benign cysts: a prospective cohort study. Fertil Steril. 2014;101(2):435-441.

5. Sugita A, Iwase A, Goto M, et al. One-year follow-up of serum antimullerian hormone levels in patients with cystectomy: are different sequential changes due to different mechanisms causing damage to the ovarian reserve? Fertil Steril. 2013;100(2):516-522 e513.

6. Kostrzewa M, Wilczyński JR, Głowacka E, Żyła M, Szyłło K, Stachowiak G. One-year follow-up of ovarian reserve by three methods in women after laparoscopic cystectomy for endometrioma and benign ovarian cysts. Int J Gynaecol Obstet. 2019;146(3):350-356. doi:10.1002/ijgo.12884

7. Durlinger AL, Visser JA, Themmen AP. Regulation of ovarian function: the role of anti-Mullerian hormone. Reproduction. 2002;124 (5):601-609. doi:10.1530/rep.0.1240601

8. Nelson SM, Anderson RA, Broekmans FJ, Raine-Fenning N, Fleming R, La Marca A. Anti-Mullerian hormone: clairvoyance or crystal clear? Human reproduction. 2012;27(3):631-636. doi:10.1093/humrep/der446

9. van Rooij IA, Tonkelaar I, Broekmans FJ, et al. Anti-mullerian hormone is a promising predictor for the occurrence of the menopausal transition. Menopause. 2004;11(6 Pt 1):601-606. doi:10.1097/01. GME.0000123642.76105.6E

10. de Vet A, Laven JS, de Jong FH, Themmen AP, Fauser BC. Antimullerian hormone serum levels: a putative marker for ovarian aging. Fertil Steril. 2002;77(2):357-362.

11. van Rooij IA, Broekmans FJ, Te Velde ER, et al. Serum anti-Mullerian hormone levels: a novel measure of ovarian reserve. Human Reproduction. 2002;17(12):3065-3071. doi:10.1093/humrep/ 17.12.3065

12. Wang HY, Quan S, Zhang RL, et al. Comparison of serum anti-Mullerian hormone levels following hysterectomy and myomectomy for benign gynaecological conditions. Eur J Obstet Gynecol Reprod Biol. 2013;171(2):368-371. doi:10.1016/j.ejogrb.2013.09.043

13. Cook CL, Siow Y, Taylor S, Fallat ME. Serum mullerian-inhibiting substance levels during normal menstrual cycles. Fertil Steril. 2000;73(4):859-861. doi:10.1016/S0015-0282(99)00639-1

14. van Disseldorp J, Faddy MJ, Themmen AP, et al. Relationship of serum antimullerian hormone concentration to age at menopause. J Clin Endocrinol Metab. 2008;93(6):2129-2134. doi:10.1210/jc.2007-2093

15. Somigliana E, Berlanda N, Benaglia L, Vigano P, Vercellini P, Fedele L. Surgical excision of endometriomas and ovarian reserve: a systematic review on serum antimullerian hormone level modifications. Fertil Steril. 2012;98(6):1531-1538. doi:10.1016/j. fertnstert.2012.08.009

16. Hwu YM, Wu FS, Li SH, Sun FJ, Lin MH, Lee RK. The impact of endometrioma and laparoscopic cystectomy on serum anti-Mullerian hormone levels. Reproduct Biol Endocrinol. 2011;9:80. doi:10.1186/ 1477-7827-9-80

17. Vignali M, Mabrouk M, Ciocca E, et al. Surgical excision of ovarian endometriomas: does it truly impair ovarian reserve? Long term anti-Mullerian hormone (AMH) changes after surgery. $J$ Obstet Gynaecol Res. 2015;41(11):1773-1778. doi:10.1111/jog.12830

18. Ding Y, Yuan Y, Ding J, Chen Y, Zhang X, Hua K. Comprehensive Assessment of the Impact of Laparoscopic Ovarian Cystectomy on Ovarian Reserve. J Minim Invasive Gynecol. 2015;22(7):1252-1259. doi:10.1016/j.jmig.2015.07.011

19. Sugita A, Iwase A, Goto M, et al. One-year follow-up of serum antimullerian hormone levels in patients with cystectomy: are different sequential changes due to different mechanisms causing damage to the ovarian reserve? Fertil Steril. 2013;100(2):516-522. doi:10.1016/j.fertnstert.2013.03.032

20. Spang A. ARF1 regulatory factors and COPI vesicle formation. Curr Opin Cell Biol. 2002;14(4):423-427. doi:10.1016/S0955-0674(02)00346-0 
21. Litta P, D’Agostino G, Conte L, et al. Anti-Mullerian hormone trend after laparoscopic surgery in women with ovarian endometrioma. Gynecol Endocrinol. 2013;29(5):452-454. doi:10.3109/ 09513590.2012 .758704

22. Wang D, Liu H, Li D, et al. Comparison of the impact of single-port laparoscopic and conventional laparoscopic ovarian cystectomy on the ovarian reserve in adult patients with benign ovarian cysts. Minim Invasive Ther Allied Technol. 2019;29(4):224-231. doi:10.1080/ 13645706.2019.1624575

23. Seyhan A, Ata B, Uncu G. The Impact of Endometriosis and Its Treatment on Ovarian Reserve. Semin Reprod Med. 2015;33 (6):422-428. doi:10.1055/s-0035-1567820

24. Salihoglu KN, Dilbaz B, Cirik DA, Ozelci R, Ozkaya E, Mollamahmutoglu L. Short-Term Impact of Laparoscopic Cystectomy on Ovarian Reserve Tests in Bilateral and Unilateral Endometriotic and Nonendometriotic Cysts. J Minim Invasive Gynecol. 2016;23(5):719-725. doi:10.1016/j.jmig.2016.02.018

25. Celik HG, Dogan E, Okyay E, et al. Effect of laparoscopic excision of endometriomas on ovarian reserve: serial changes in the serum antimullerian hormone levels. Fertil Steril. 2012;97(6):1472-1478. doi:10.1016/j.fertnstert.2012.03.027

26. Uncu G, Kasapoglu I, Ozerkan K, Seyhan A, Oral Yilmaztepe A, Ata B. Prospective assessment of the impact of endometriomas and their removal on ovarian reserve and determinants of the rate of decline in ovarian reserve. Human Reproduction. 2013;28 (8):2140-2145. doi:10.1093/humrep/det123

27. Biacchiardi CP, Piane LD, Camanni M, et al. Laparoscopic stripping of endometriomas negatively affects ovarian follicular reserve even if performed by experienced surgeons. Reprod Biomed Online. 2011;23 (6):740-746. doi:10.1016/j.rbmo.2011.07.014

28. Iwase A, Nakamura T, Kato N, et al. Anti-Mullerian hormone levels after laparoscopic cystectomy for endometriomas as a possible predictor for pregnancy in infertility treatments. Gynecol Endocrinol. 2015;1-5.

29. Urman B, Alper E, Yakin K, et al. Removal of unilateral endometriomas is associated with immediate and sustained reduction in ovarian reserve. Reprod Biomed Online. 2013;27(2):212-216. doi:10.1016/j.rbmo.2013.04.016

30. Raffi F, Metwally M, Amer S. The impact of excision of ovarian endometrioma on ovarian reserve: a systematic review and meta-analysis. J Clin Endocrinol Metab. 2012;97(9):3146-3154. doi:10.1210/jc.2012-1558
31. Cook CL, Siow Y, Brenner AG, Fallat ME. Relationship between serum mullerian-inhibiting substance and other reproductive hormones in untreated women with polycystic ovary syndrome and normal women. Fertil Steril. 2002;77(1):141-146. doi:10.1016/ S0015-0282(01)02944-2

32. Iwase A, Hirokawa W, Goto M, et al. Serum anti-Mullerian hormone level is a useful marker for evaluating the impact of laparoscopic cystectomy on ovarian reserve. Fertil Steril. 2010;94(7):2846-2849. doi:10.1016/j.fertnstert.2010.06.010

33. Kitajima M, Khan KN, Hiraki K, Inoue T, Fujishita A, Masuzaki H. Changes in serum anti-Mullerian hormone levels may predict damage to residual normal ovarian tissue after laparoscopic surgery for women with ovarian endometrioma. Fertil Steril. 2011;95 (8):2589-2591. doi:10.1016/j.fertnstert.2011.01.036

34. Mohamed ML, Nouh AA, El-Behery MM, Mansour SA. Effect on ovarian reserve of laparoscopic bipolar electrocoagulation versus laparotomic hemostatic sutures during unilateral ovarian cystectomy. Int J Gynaecol Obstet. 2011;114(1):69-72. doi:10.1016/ j.ijgo.2011.01.010

35. Dogan E, Ulukus EC, Okyay E, Ertugrul C, Saygili U, Koyuncuoglu M. Retrospective analysis of follicle loss after laparoscopic excision of endometrioma compared with benign nonendometriotic ovarian cysts. Int J Gynaecol Obstet. 2011;114(2):124-127. doi:10.1016/j.ijgo.2011.04.002

36. Shao MJ, Hu M, He YQ, Xu XJ. AMH trend after laparoscopic cystectomy and ovarian suturing in patients with endometriomas. Arch Gynecol Obstet. 2015;293(5):1049-1052. doi:10.1007/s00404015-3926-4

37. Sahin C, Akdemir A, Ergenoglu AM, et al. Which Should Be the Preferred Technique During Laparoscopic Ovarian Cystectomy. Reprod Sci. 2017;24(3):393-399. doi:10.1177/1933719116657195

38. Song T, Kim WY, Lee KW, Kim KH. Effect on ovarian reserve of hemostasis by bipolar coagulation versus suture during laparoendoscopic single-site cystectomy for ovarian endometriomas. J Minim Invasive Gynecol. 2015;22(3):415-420.

\section{Publish your work in this journal}

The International Journal of Women's Health is an international, peerreviewed open-access journal publishing original research, reports, editorials, reviews and commentaries on all aspects of women's healthcare including gynecology, obstetrics, and breast cancer. The manuscript management system is completely online and includes a very quick and fair peer-review system, which is all easy to use. Visit http://www.dovepress.com/testimonials.php to read real quotes from published authors. 